\title{
Die Ideenmaschine
}

Erhard Taverna

Mit Inspirationen ist es so eine Sache. Gute Einfälle sind rar, Geistesblitze noch seltener und mit den Musenküssen ist es wie mit der Liebe, man kann sie nicht erzwingen. In vorindustriellen Zeiten verwöhnten Zeus und Mnemosyne Wissenschaft und Kunst mit ihren geflügelten neun Töchtern. Die Überheblichen mochten sie allerdings nicht. Wer allzu anmassend forderte, riskierte sein Gedächtnis, wurde geblendet, gerupft oder in eine Dohle verwandelt. Heute straft nur noch der Markt, denn gute Ideen sind käuflich, zumindest für jene Branchen, die unsere Konsummaschine am Laufen halten. In den letzten Jahren haben sich zahlreiche Firmen etabliert, die ihre Kunden mit Ideen und Erfindungen beliefern. Sie nennen sich Ideenfabriken, Ideenlabors, Think Tank, Creaholic, Innovation Workshop oder BrainStore. Die Produktionstechniken des «Idea Engineering» werden kommerziell angeboten und in zahlreichen Forschungsprojekten weiterentwickelt.

Zwei Studenten und eine Gymnasiastin haben 1989 die Firma BrainStore in Biel gegründet. Heute ein Betrieb mit 60 festangestellten Mitarbeitern und rund 5000 weltweit tätigen Temporären. Kunden sind Grossfirmen wie Novartis, UBS, SBB, Radio DRS und Nestlé, aber auch das Bundesamt für Gesundheitswesen, Krankenkassen, kleine Unternehmen und Privatpersonen. Ob Coca-Cola, ein Snackprodukt, eine Hepatitis-B-Impfung oder die Stop-Aids-Kampagne, für alle diese Aufträge wurden Ideen nach industriellen Methoden entwickelt. Dabei liefert jeder Findungsprozess 10 bis 60 umsetzungsreife Ideen über sechs Etappen, die von der 34jährigen Journalistin und Mitgründerin Nadja Schnetzler in ihrem Buch «Die Ideenmaschine» präzis und unterhaltsam beschrieben werden. Das Bild der Maschine steht für eine ausgeklügelte Organisation, die nach einem festen Ablaufschema mit definierten Werkzeugen arbeitet. Nach dem Prinzip Beschaffen - Verdichten - Auswählen wird die Produktion minutiös gesteuert, in getrennte Phasen zerlegt und wieder zusammengebaut. Der Erfolg beginnt mit der richtigen Mischung der «CreatingCommunity» aus Insidern und Outsidern. BrainStore hat das Einbeziehen von extremen Blickwinkeln einander völlig fremder Welten perfektioniert. Das Spektrum reicht von Teenagern über Studenten und Haus-

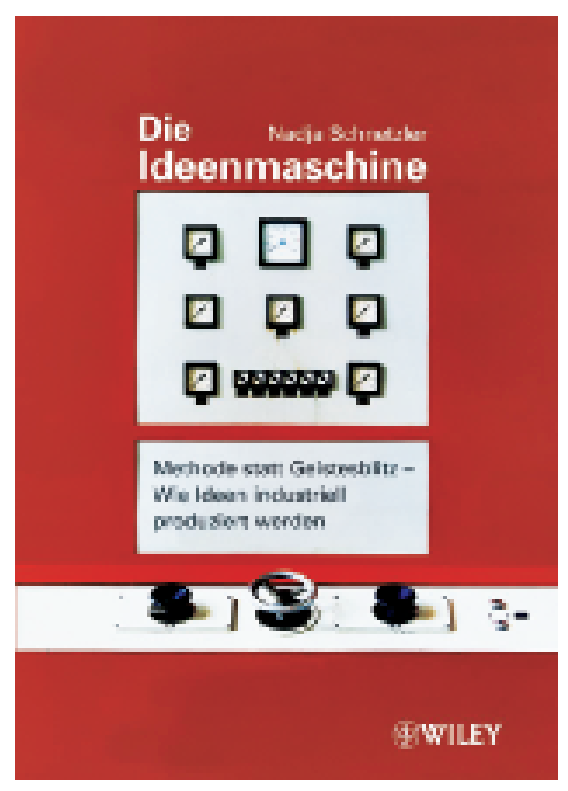

frauen zu Ingenieuren, Professoren und Experten für jegliche Fachgebiete. Die Teilnahme von Jugendlichen gehört zum Verfahren. Sie seien erfrischend frech und ungezwungen, ehrlich und direkt, rasch, effizient und spontan. Nicht zuletzt würden sie eine neue Generation vertreten, die mit neuen Technologien, Marketing und Werbung vertrauter sei als viele Entscheidungsträger. Aus dem gut funktionierenden Netzwerk werden die Spezialisten und Querdenker für das erste Team zusammengesucht. Ausstattung der Teilnehmer, Pausen und Erfrischung, Einrichtung der Räumlichkeiten, Techniken des Brainstormings und der nötige Zeitdruck, nichts bleibt dem Zufall überlassen. Fragebogen, Interviews, TrendScouting und NetScouting gehören zu den Schlüsseltechniken der ersten Phase. Die Verdichtung geschieht in einem ersten Schritt in der «IdeaCity», der virtuellen Ideenstadt, wo der Ideentopf von rund 3000 Einfällen auf 150200 Ideen konzentriert wird. In einem zweiten Schritt wird entschieden, was im Topf bleibt, rausgeworfen oder abgeändert wird. Ideen werden bewertet und kundengerecht aussortiert, bis 10 oder 20 in einem letzten Gang ihre Form erhalten. Dem Verdichten folgt abschliessend ein diszipliniertes Auswahlverfahren nach zwingenden Spielregeln, in das der Kunde miteinbezogen ist. Den inhaltlich optimalen und zeitgenauen 
Ablauf organisiert das Ideenmanagement. Es sind immer zwei Personen für den Kunden zuständig: Der Ideendirektor ist der Chef des Projekts, er führt die Gespräche und Verhandlungen und empfiehlt am Ende eine oder mehrere Ideen für die Umsetzung. Der Projektmanager begleitet den Kunden durch das Projekt und überwacht die Produktion. Das ganze System von 16 frei kombinierbaren Basisverfahren wird von Nadja Schnetzler gut verständlich und humorvoll vorgestellt und mit vielen Beispielen illustriert. BrainStore unterstützt auch Unternehmen beim Einrichten firmeninterner Ideenfabriken.

Ein originelles Angebot ist die BrainParty, «der Ideenfindungsworkshop mit garantierten Resul- taten, kombiniert mit einer rauschenden Party. Das Ereignis für langweilige Innovationskultur, für Teams, die dringend einen Kick brauchen». Das Angebot verspricht nach spätestens 7-20 Stunden handfeste, sofort realisierbare Ideen, die allen geforderten Kriterien entsprechen. Jeder lustlosen Verwaltung und jedem müden Spitaloder Praxisteam dringend empfohlen.

- Nadja Schnetzler. Die Ideenmaschine. 5. Auflage. Weinheim: Wiley-VCH; 2006. Taschenbuch, 228 Seiten.

- BrainStore, Aarbergstrasse 3-5, Postfach 336, 2501 Biel, Tel. 03232100 00, www.brain store.com. 